\title{
Histoires de vie avec l'alternance: la voie de recherche- formation en deux temps, trois mouvements et le master formation et développement durable au Brésil
}

\author{
Gaston Pineau ${ }^{1}$, Pedro Puig-Calvó ${ }^{2}$ \\ ${ }^{1}$ I'Université de Tours - France. Science de l'éducation. 0 rue du Plat D'Etain 37020 Tours cedex 1, Paris, France / Universidad \\ du Québec. 405 Rue Sainte-Catherine Est, Montréal, QC H2L 2C4, Montreal, Canada. ${ }^{2}$ Universitat Internacional de Catalunya \\ - UIC / Association Internationale des Mouvements Familiaux de Formation Rurale - AIMFR, Spain. \\ Author for correspondence: gaston.pineau@gmail.com
}

RÉSUMÉ. La communication voit l'anniversaire de ces 50 ans de l'alternance au Brésil, comme un moyen d'opérer une double boucle réflexive sur deux histoires de vie socio-professionnelle avec l'alternance. Elle commence par un survol historique principalement francophone, d'une voie de recherche-formation de l'alternance comme synchroniseur éco-social de temporalités multiples en deux temps (expérientiel/formel) et trois mouvements (auto, socio, écoformation). La deuxième partie expose la philosophie éducative d'un master international Formation et développement durable (2002-2004), construit avec et pour les professionnels de l'alternance au Brésil. Elle présente les 17 mémoires produits selon une structure quadripolaire de l'alternance, croisant deux finalités développement de la personne et du milieu - avec deux moyens principaux - une pédagogie spécifique et une organisation partenariale. La conclusion évoque l'horizon d'une compétence rythmique existentielle à apprendre par une attention plus grande aux rythmes écologiques qu'aux cours de la bourse, des maîtres et contremaîtres.

Mots clés: Alternance, Formation Expérientielle, Formation Formelle, Autoformation, Socioformation, Écoformation, Développement Durable, Devenir Personnel, Devenir Social, Devenir Écologique, Synchroniseur.

\begin{tabular}{|l|l|l|l|l|l|l|} 
RBEC & Tocantinópolis/Brasil & v. 4 & e7279 & $10.20873 /$ uft.rbec.e7279 & 2019 & ISSN: 2525-4863 \\
\hline
\end{tabular}




\section{Historias de vida con alternancia: la trayectoria de investigación-entrenamiento en dos pasos, tres movimientos y el máster de formación y desarrollo sostenible en Brasil}

RESUMEN. La comunicación concibe el aniversario de estos 50 años de alternancia en Brasil, como una forma de operar un doble bucle reflexivo en dos historias de la vida socioprofesional con la alternancia. Comienza con una visión histórica principalmente francófona de una ruta de investigación-formación de la alternancia como un sincronizador eco-social de múltiples temporalidades en dos etapas (experiencial/formal) y tres movimientos (auto, socio, eco-formación). La segunda parte expone la filosofía educativa de un máster internacional de Formación y Desarrollo Sostenible (2002-2004), construido con y para los profesionales de la alternancia en Brasil. Presenta las 17 memorias producidas según una estructura cuadripolar de la alternancia, cruzando dos finalidades - el desarrollo de la persona y el medio ambiente con los dos medios principales - una pedagogía específica y una organización de colaboración: la asociación. La conclusión evoca el horizonte de una competencia rítmica existencial que aprenderemos prestando más atención a los ritmos ecológicos que a la cotización de la bolsa o a los que toman decisiones.

Palabras clave: Alternancia, Formación Experiencial, Formación Formal, Autoformación, Socio-Formación, Ecoformación, Desarrollo Sostenible, Transformación Personal, Transformación Social, Transformación Ecológica, Sincronizador. 


\title{
Stories of life with alternating cycle education: the trajectory of research-training in two steps, three movements and the master of training and sustainable development in Brazil
}

\begin{abstract}
The communication sees the anniversary of these 50 years of alternating cycle pedagogy in Brazil, as a means of operating a double reflective loop on two stories of socioprofessional life with work-study. It begins with a mainly French-speaking historical overview of a research-training path of work-study as an eco-social synchronizer of multiple temporalities in two stages (experiential/formal) and three movements (auto, socio, eco-training). The second part presents the educational philosophy of an international master's in training and Sustainable Development (2002-2004), built with and for professionals in alternating cycle pedagogy in Brazil. It presents the 17 dissertations produced according to a quadrupole alternating cycle structure, crossing two purposes - development of the person and the environment - with two main means - a specific pedagogy and a partnership organization. The conclusion evokes the horizon of an existential rhythmic skill to learn by paying more attention to ecological rhythms than to the price of the stock market or to those who make decisions.
\end{abstract}

Keywords: Alternating Cycle, Experiential Training, Formal Training, Self-Training, Socio-Training, Eco-Training, Sustainable Development, Personal Transformation, Social Transformation, Ecological Transformation, Synchronizer. 


\section{Introduction}

Il faut être reconnaissant aux concepteurs de ce numéro de ponctuer ces 50 ans de l'alternance au Brésil. En effet c'est d'abord souligner cette date comme importante historiquement. Et c'est permettre d'opérer une boucle réflexive sur ce qu'en disent les recherches nationales et internationales. Double force, récursive et propulsive, des remémorations pour construire une histoire spécifique en apprenant à articuler de façon singulière passé, présent et futur. Marquer, et même fêter un anniversaire, est un synchroniseur majeur pour reconnaître à part entière et articuler des moments, des lieux, des personnes dont la variété et la complémentarité des rôles n'apparaissent souvent qu'avec un certain recul. Et plus ces apports sont originaux et sont partis des lointaines périphéries sociales, plus les risques d'oubli et de refoulement temporel sont grands.

C'est le cas de l'alternance qui a germé petitement en milieu rural profond en opposition au modèle scolaire dominant internationalement depuis presque deux siècles. Pouvoir fêter les 50 ans au Brésil et les plus de 80 ans dans le monde francophone de ce mouvement sociohistorique de recherche-formation d'alternatives éducatives est déjà tout un événement en soi. Et je me trouve privilégié de pouvoir y participer. Je le ferai en deux temps. En opérant d'abord un survol d'une voie de conceptualisation de l'alternance pour en expliciter les principaux acquis. Puis en revenant sur l'expérience exploratoire d'un master international au Brésil qui, en 2002-2004, a voulu construire une ingénierie de recherche-formation universitaire par alternance pour les professionnels de l'alternance.

Survol historique de la voie de recherche-formation de l'alternance en deux temps (expérientiel/formel) et trois mouvements (auto/socio/éco formation)

L'apprentissage de l'alternance est au cœur de ma formation permanente du cours de ma vie, même si j'ai dû attendre 35 ans, avant de faire éclater consciemment ma représentation vécue d'un cours linéaire et irréversible des âges de la vie: études, travail, retraite. Mais ensuite, mon recrutement universitaire sur un poste profilé Alternance et éducation des adultes m'a entraîné à entreprendre coopérativement des couplages structurels entre universités et centres d'alternance pour construire des diplômes de production de savoirs plus que de consommation de cours, en France d'abord avec le Centre National Pédagogique des MFR, et au Brésil avec L'AIMFR et les associations brésiliennes. Mon principal coach 
international est toujours vivant: Pedro Puig-Calvó. Les autres sont morts en grande partie: Daniel Chartier, Jacques Legroux, Georges Lerbet, sauf JeanClaude Gimonet. Mais ils sont encore très inspirants. On les retrouvera dans le second temps de ma communication sur mon apprentissage d'une ingénierie internationale d'alternance

formative/transformative avec

L'Association Internationale des Mouvements Familiaux de Formation Rurale (AIMFR) et la conception et conduite coopérative du master international Formation et développement durable au Brésil (2002-2004).

\section{Années 60: premier moment d'initiation obscure par rupture expérientielle éprouvante avec les formations formelles}

Né en milieu rural bas-breton en France, je suis le premier du village à avoir fait des études secondaires. Après avoir passé mon bac en obéissant à la prudente injonction «Passe ton bac d'abord, tu verras après», j'ai décroché volontairement pour ne pas consommer une rupture culturelle avec mon milieu d'origine. Je suis devenu ouvrier agricole. Au bout de cinq ans, la charge socio-cognitive non exprimée de la formation expérientielle acquise a été telle, qu'elle m'a amené avec d'autres décrocheurs comme moi, à m'inscrire à l'université, d'abord en psychologie, puis en sciences de l'éducation émergentes en France en 1967. Les vagues de 1968 et de l'an 1 (1971) de la formation continue dans le cadre de l'éducation permanente ont orienté mon passage en apesanteur entre la planète étude et la planète travail, selon le modèle planétaire des étapes de vie au travail de Danielle RiverinSimard (1984). Les nouvelles professionnalités alors en émergence ont assuré un nouvel atterrissage plus porteur d'avenir sur la planète travail: conseiller d'orientation continue d'adultes dans le bassin minier lorrain en reconversion puis, responsable de recherche au service de l'éducation permanent, qui devient Faculté 1975, de L’Université de Montréal.

Mais jusque dans les années 76, l'alternance ne me disait rien; elle restait pour moi un gros mot étranger. Il a fallu l'interview non prévu d'une journaliste de l'Association de formation professionnelle des adultes (AFPA) enquêtant sur ce sujet pour m'y éveiller. Elle avait entendu parler de moi comme un cas possiblement intéressant pour son enquête. Comme on ne savait pas trop comment commencer l'entretien, elle me dit: «Raconte donc ta vie, on va voir ce qu'on peut en tirer pour l'alternance». En fait est remontée alors de mes 35 ans, la période la plus éprouvante 
de ma première insertion socioprofessionnelle entre 20 et 25 ans, avec guerre d'Algérie comprise. Une heure et demie d'entretien oral enregistré, à la fin duquel elle me dit: «Je ne vois pas trop ce que je peux en faire. Et je n'ai pas le temps de réécouter. Peux-tu m'en faire une synthèse écrite».

Je reviens au Québec avec cette commande. Et quinze jours après, je lui envoie cinq pages, très laborieusement écrites. Par retour du courrier, elle me répond: «Impubliable. La vie n'est pas si noire que ça. Et une publication t'exposerait trop et te rendrait trop vulnérable». Très déçu, je remise mes 5 pages dans un tiroir. Mais leur titre me reste en mémoire et me travaille. Je les avais intitulées: Autoformation et quotidienneté. C'est ainsi que je nommais ce qui est reconnu depuis comme l'éducation informelle de la vie quotidienne, celle qui peut surgir entre l'éducation formelle du système scolaire et l'éducation non formelle des autres organisations, entre autres du travail.

Cet «entre» informel du quotidien, revient de façon permanente, $24 \mathrm{~h}$ sur 24 , au moins sous deux formes répétitives et cycliques très différentes, voire contraires: le jour/la nuit; le diurne/le nocturne. C'était le vécu de la forme la plus obscure, la nuit, qui était remontée, dans toute son opacité dissolvant les formes du jour dans justement un informel angoissant, plongeant dans «un état d'anté-sujet» comme dit Bachelard, grand explorateur du «travail alterné des images et des concepts, deux bonnes consciences qui seraient celle du plein jour, et celle qui accepte le côté nocturne de l'âme» (Bachelard, 1963).

Mais la conquête d'une alternance intégrative de ces deux espaces/temps matériels et symboliques contraires, suppose un triple apprentissage: celui de la spécificité formative de chacun de ces deux espaces/temps de vie contraire et ensuite de leur reliance à rythmer selon une mise ensemble, en sens, en forme, selon une dialectique de formation d'une durée, d'une histoire personnelle. Je n'en étais qu'au premier, découvrant l'existence de la face nocturne de la vie quotidienne et le désapprentissage nécessaire des formes du jour pour l'entamer. Pas étonnant que mes débuts d'expression soient jugés négatifs et socialement dangereux pour moi.

\section{Années 70-90 : deuxième et troisième moment d'essai de mise en mots, en concepts, en sens, de l'expérience vécue}

Il m'a fallu beaucoup de temps de retour réflexif sur cette expérience d'espace/temps nocturne pour commencer à en arracher quelques formulations quelques mises en forme - plus communicables. Ce second et troisième 
temps d'initiation fut rendu possible grâce l'éclairage conceptuel apporté par la découverte de deux théorisations éducatives bien antérieures à l'institutionnalisation de l'école au 19ème 20ème siècle. Il s'agit en effet du mythe de la caverne de Platon du 4ème siècle avant notre ère; et de la théorie des trois maîtres éducatifs de Rousseau - soi, les autres et les chose - fin du 18ème.

\section{Le mythe de la Caverne et les deux temps - expérientiel/formel - de la formation permanente}

Platon développe ce mythe dans un livre de politique - La République, Livre 5 - pour former des gouvernants philosophes, amis de la sagesse, à la place des guerriers les plus violents. L'éducation courte l'Académie - que Platon avait d'abord essayée, montrait déjà ses limites. Il imagine et conçoit alors sous la forme du Mythe de la caverne ce qu'il appelle «le long circuit éducatif préfigurant la formation permanente actuelle (Pineau, 1977).

Ce mythe modélise ce long circuit éducatif du cours de la vie comme une alternance nuit/jour symbolisée par un ancrage pratique sur terre dans l'espace semi-obscur d'une caverne - symbole de l'utérus d'où nous venons et de la tombe où nous allons - et la lumière extérieure du grand jour apportée par le soleil. La formation de chaque vivant est prise avec ces deux pôles nuit/jour alternant quotidiennement. Cette alternance quotidienne nuit/jour constitue l'environnement matriciel écologique et symbolique d'autres types de polarisations bio-cognitives. L'école s'est institutionnalisée le jour, associant l'éducation au monde lumineux des idées claires, distinctes, précises, structurées devant éclairer le reste: la pratique, le vécu, l'action, le sensible refoulé dans la nuit de l'esprit.

$\mathrm{La}$ trilogie relativement récente d'éducation formelle/non formelle/informelle est déjà une grande avancée en faisant sauter la superposition dichotomique du jour comme école contre nuit à éduquer. Mais elle le fait de façon privative, avec les lunettes formelles de l'éducation du jour, ne voyant pas les formes de ce qui se passe la nuit. Elle reconnaît que ça peut être éducatif, mais ne peut le nommer qu'avec les préfixes privatifs - non ou in -. La montée ces dernières années du terme de formation expérientielle est indicatrice de la dimension formatrice possible originale et à part entière de ce qui se passe à l'intérieure de la caverne même si on y est vitalement attaché, en contact direct. C'est en clair-obscur, comme des formes mouvantes sur des écrans géants. C'est 
alors que prend tout son sens dialectique l'alternance entre action/réflexion; pratiques/théories; parcours/discours; mondes clairs obscurs d'expériences sensibles peu compréhensible/mondes lumineux des idées intelligibles; et même monde sensible féminin/monde rationnel masculin. Les contraires se confrontent existentiellement selon une dialectique créatrice de sens multiples.

C'est à la compréhension de la formation opérée par la rencontre dialectique de ces deux temps expérientiels/formels - qu'aide la théorie de trois maîtres en éducation existentielle de Jean-Jacques Rousseau.

\section{Les trois maîtres de l'éducation existentielle de Jean-Jacques Rousseau}

Dès les premières pages de l'Émile ou De l'éducation (Rousseau, 2009) pose très clairement que ce qui rend l'éducation aussi complexe que la vie, c'est qu'elle dépend de trois maîtres: soi (sa nature individuelle, ses dispositions primitives), les autres et les choses. Et en plus les leçons de ces trois maîtres sont souvent différentes et même opposées et conflictuelles (Pineau, 2015). L'aide des préfixes - auto, socio et éco - m'ont beaucoup aidé à construire une théorie tripolaire des formations vitales et expérientielles (Pineau, 2004) pour travailler les trois mouvements de l'alternance, entre soi, les autres et les choses:

- Le préfixe auto a été pris comme foyer de théorisation des pratiques émancipatoires et autonomisantes de la formation, dépendant au départ des autres et des choses. L'ouvrage L'autoformation. Perspectives de recherche (Carré, Moisan $\&$ Poisson, 2010) donne les perspectives de recherche actuelles.

- Le préfixe socio a permis de travailler cette dépendance sociale. Il s'est dédoublé progressivement en hétéroformation, pour indiquer le pouvoir de formation de personnes ayant un statut hiérarchique supérieur à soi; et en coformation, pour prendre en compte les interactions et transactions de parité, de réciprocité, d'intimité entre pairs: ami, compagnon, camarade, conjoint (Eneau, 2005).

- Et enfin le préfixe éco a permis d'essayer de résonner aux choses qui ne parlent pas le même langage que nous et d'explorer la difficile transformation des rapports d'usage avec elles en rapports plus sages ecoformation (Pineau, \& Galvani, 2017).

Début des années 2000: Théorisation de l'alternance, comme synchroniseur social et écologique en deux temps trois mouvements 
J'ai voulu ouvrir les années 2000 avec un ouvrage tentant de synthétiser mes recherches antérieures sur les temps et contretemps en éducation permanente : Temporalités en formation. Vers de nouveaux synchroniseurs (Pineau, Temporalidades na formaçao. Rumbo a novos sincronizadores, 2004). Forgé par les chronobiologistes dans les années 80, comme «tout facteur dont les variations périodiques sont susceptibles de modifier la période et/ou la phase d'un rythme biologique (Reinberg \& Ghata, 1982) le terme de synchroniseur a surtout été repris en électronique, automobile, informatique, audiovisuel. Et peu dans les sciences humaines et sociales du temps, qui en sont surtout restés à l'analyse des mouvements de synchronisation/désynchronisation rythmique, sans aller à isoler dans cette complexité effervescente des synchroniseurs ou des donneurs de temps clefs, capable de contrebalancer la loi horaire homogénéisante d'un temps qui se veut universel. Cet ouvrage s'y essaie avec deux pratiques courantes dont la prise en compte réflexive à part entière peut déployer et actualiser un potentiel inouï de construction de sens: raconter sa vie comme synchroniseur linguistique; et alterner comme synchroniseur écologique.

La complexité de la prise en compte sociale et personnelle de ce synchroniseur écologique a pu être modélisée selon une matrice des alternances possibles entre les deux temps expérientiels/formels et les trois mouvements de personnalisation, de socialisation et d'écologisation.

Tableau 1 - Matrice des alternances possibles entre les deux temps et les trois mouvements de la formation.

\begin{tabular}{|l|l|l|l|}
\hline \multicolumn{1}{|c|}{ Temps } & \multicolumn{2}{|c|}{ Mouvements } \\
\hline & Personnalisation & Socialisation & Écologisation \\
\hline De formation formelle & & & \\
\hline De formation expérientielle & & & \\
\hline
\end{tabular}

Source: (Pineau, G. Temporalidades na formaçao. Rumbo a novos sincronizadores, 2004).

La vision dominante de l'alternance la réduit souvent à une alternance études/travail, les études représentant le temps de la formation formelle et le travail celui de la formation non formelle et informelle. Cette vision restreinte a un besoin urgent de s'élargir par un déverrouillage des pouvoirs hétéronomes pour une reconnaissance à part entière des pôles auto, co et écoformateurs s'autonomisant dans ces formations expérientielles, hors des autorités formelles immédiates des maîtres et contre-maîtres.

La reconnaissance du pôle éco appelle, pour tous les acteurs, un déverrouillage de la sensibilité humaine 
aux éco et aux biorythmes à laquelle l'agroécologie montante

est particulièrement éveillée et attentive (Moneyron, 2018) L'alternance études/travail s'enracine, même de façon encore peu conscientisée, dans une alternance environnementale écoformatrice macro et microcosmique. En 2017, le prix Nobel de médecine attribué à trois chercheurs en chronobiologie consacre scientifiquement l'influence des rythmes circadiens sur nos comportements. La jonction entre les biorythmes et la montée de la mobilisation écologique actuelle est à faire. (Lesourd, 2009) étudie comment une culture polyphasique - attentive aux différentes phases de conscience expérienciées au cours de la journée «favoriserait chez le sujet une conscientisation fine de ses propres vécus microliminaires et, partant, une formation plus fine de l'attention exploratrice de ces vécus». Ainsi s'expliciteraient mieux les processus de transformations existentielles et de développement durable.

\section{Années 2010 - 2020: émergence d'un nouvel âge pour l'alternance?}

Ces dernières années ont été ponctuées par une série d'événements et de production qui semble préfigurer un nouvel âge de l'alternance. En 2017, un colloque à L'Université de Sherbrooke L'alternance, formation et transformation a permis de faire le point. Après deux numéros relativement récents de la revue Éducation Permanente sur l'alternance, au-delà du discours (2012-1), et l'alternance, du discours à l'épreuve (2012-4), un autre au Québec, de Phronésis 2016, no3 et 4, "Les reliances de L'alternance en formation et ses effets sur les processus de construction identitaire des alternants" et ce numéro anniversaire au Brésil, on a l'impression d'un lever de soleil officiel sur l'alternance, après de longues aubes émergentes (Chartier, 2004), aux lisières des cultures rurales et des apprentissages professionnels. C'est comme si les pressions extérieures des crises écologiques et éducatives la sortaient d'un refoulement culturel moderne bicentenaire.

Un nouvel âge semble s'annoncer effervescent, gros d'enjeux socio-éducatifs, mais aussi politiques et économiques: l'alternance comme construction d'alternatives coopératives d'apprentissage pour la formation d'une ère planétaire viable et durable pour tout le monde, ou l'alternance comme nouvelle articulation stratégique des maîtres et contremaîtres pour renforcer leurs pouvoirs de reproduction? (Pineau, 2017).

Cette dans cette dynamique qu'a été conçu et réalisé, par et pour les 
professionnels de l'alternance au Brésil, le master international expérimental Formation et développement durable.

Survol de la philosophie éducative et des productions du master international formation et développement durable au Brésil (2002-2004)

Ce diplôme international en sciences de l'Éducation «Formation et Développement Durable» est le produit d'un partenariat de 10 ans de recherche et de formation sur les problèmes de liaison entre éducation et développement, entre L'Université de Tours, l'Université Nouvelle de Lisbonne et l'Union nationale des Maisons familiales rurales d'éducation et d'orientation (UNMFREO). En 1999, ce partenariat s'est élargi à l'Union nationale des écoles familiales agricoles du Brésil (UNEFAB) et à l'association Solidarité Internationale des Mouvements Familiaux de Formation Rurale (SIMFR - Belgique), grâce au premier Séminaire international sur la pédagogie de l'alternance organisé à Salvador de Bahia par les Universités Fédérale et d'État de Bahia et les organismes précités. Au début de 2001, la première promotion de vingt Brésiliens de 12 États a inauguré la première session de 15 jours à Vitoria. Ensuite, la démarche de formation-action-recherche par alternance s'est déroulée par sessions de 15 jours dans les capitales de différents états - Salvador,
Brasília (2 fois), Sao Luis, São Paulo, Florianópolis, Belo Horizonte - avec rencontre dans les universités de chaque état.

La fin de la première année a été ponctuée par un second séminaire international sur la pédagogie de l'alternance et le développement durable à Brasilia (Novembre 2002) où les étudiants ont pu communiquer publiquement l'état d'avancement de leur projet de recherche. Les soutenances eurent lieu à Brasilia en mai 2004. Et en 2009, les principaux acquis de cette formation par production de savoirs par transformation des problèmes d'action en projets de recherche, ont pu être explicités et socialisés dans l'ouvrage collectif «Alternatives socio-éducatives au Brésil. Expérience d'un master international » (Pineau, Bacherart, Couceiro, Gimonet, \& Puig-Calvó, 2009). Ce qui permet la présentation suivante de sa philosophie éducative et de ses productions.

\section{Philosophie éducative du diplôme: finalité, épistémologie et pédagogie}

La philosophie socio-éducative de ce diplôme se résume ainsi: La finalité est 1 . de construire des devenirs durables 2 . en visant à relier de façon inter et transdisciplinaire formation, action et recherche 3. par une pédagogie 
d'alternances coopératives de production de savoirs. Le tableau 1 détaille les trois points de cette proposition.

\section{Une finalité anthropoformative complexe mais vitale: construire des devenirs durables}

Le terme de «développement durable» dans le titre du diplôme indique la finalité à poursuivre par la formation qui n'est alors qu'un moyen. Finalité complexe proposée comme vitale par le rapport Brundtland. «Notre futur commun» de la Commission des Nations Unies sur l'Environnement et le Développement (CNUED) en 1987: «Mode de développement qui répond aux besoins des générations présentes sans compromettre la capacité des générations futures à répondre à leurs propres besoins». Le Premier Sommet de la Terre à Rio (1992) a énoncé des conditions concrètes. Depuis, la précision de cette large définition - et c'est son intérêt - a ouvert tout un champ de recherches, de formation et de débats interrogeant les termes même de développement durable. Les indicateurs technico-économiques occidentaux du développement plombent tellement la notion pour certains qu'il faut rompre avec elle: «Durable ne fait que tempérer le développement par considération du contexte écologique» $\quad . . \quad$ Le développement, universaliste, constitue un mythe typique $\mathrm{du}$ sociocentrisme occidental, un moteur d'occidentalisation forcenée, un instrument de colonisation des «sous-développés» (le Sud) par le Nord ... Le terme de développement durable ou soutenable peut ralentir ou atténuer mais non modifier ce cours destructeur.

$$
\text { «Il s'agit dès lors non tant de }
$$

ralentir ou d'atténuer, mais de concevoir un nouveau départ» (Morin, Au-delà de la globalisation et du développement, societémonde ou empire-monde?, 2002). Morin propose donc de rompre avec le terme et de lui substituer celui d'une politique de l'humanité: anthropolitique avec une réforme radicale de tous les systèmes d'éducation. Nous entrons dans ce débat en proposant comme finalité de ce diplôme inter-transdisciplinaire de sciences de l'éducation: construire des devenirs durables: une finalité anthropoformative complexe mais vitale. Nous rejoignons la seconde génération de Paolo Freire qui prend la Terre-Patrie comme paradigme d'apprentissage pour lutter contre une mondialisation compétitive clairement insoutenable à long terme «Pedagogia da Terra» (Gadotti, 2000) offre une vision altermondialiste les plus construites actuellement.

En remplaçant «développement» par «devenirs», nous nous délestons d'une 
vision développementaliste trop idéologique, et nous renforçons une approche temporelle constructiviste pointée par le terme de durable. L'humanité n'est pas une donnée tout faite, mais une construction en devenirs permanents à la durée aléatoire et incertaine. La formation de l'humanité l'anthropoformation - est donc aux prises avec une quête et une conquête spatiotemporelle à mener en permanence en essayant de conjuguer deux extrêmes pour exister : instant et durée. Ni l'un, ni l'autre ne sont assurés, pas plus que leur liaison réciproque. Advenir à son devenir et s'y maintenir oblige à apprendre à conjuguer à la première personne du singulier et du pluriel des temps et contretemps physiques, physiologiques et sociaux.

Cet apprentissage spatio-temporel exige lui-même des temps spécifiques et l'alternance peut être vue comme un moyen, sinon le moyen majeur de synchroniser des temps contraires. De façon très pragmatique les deux premiers séminaires internationaux sur l'alternance qui ont ponctué fortement le diplôme, ont commencé à expliciter le potentiel de cette alternative socio-éducative. Voici la Philosophie du master international: Formation et développement durable» et ses productions.

Tableau $\mathrm{n}^{\circ} 2$ - Philosophie du master international: «Formation et développement durable».

\begin{tabular}{|c|}
\hline $\begin{array}{l}\text { 1. Construire des devenirs durables: une finalité } \\
\text { anthropoformative complexe mais vitale. } \\
1.1-\text { Par une approche temporelle } \\
\text { constructiviste. } \\
1.2-\text { Des trois piliers du développement } \\
\text { durable: personnel, socio-économique et } \\
\text { écologique. } \\
1.3-\quad \text { A conjuguer spatio-temporellement de } \\
\text { façon transgénérationnelle. }\end{array}$ \\
\hline 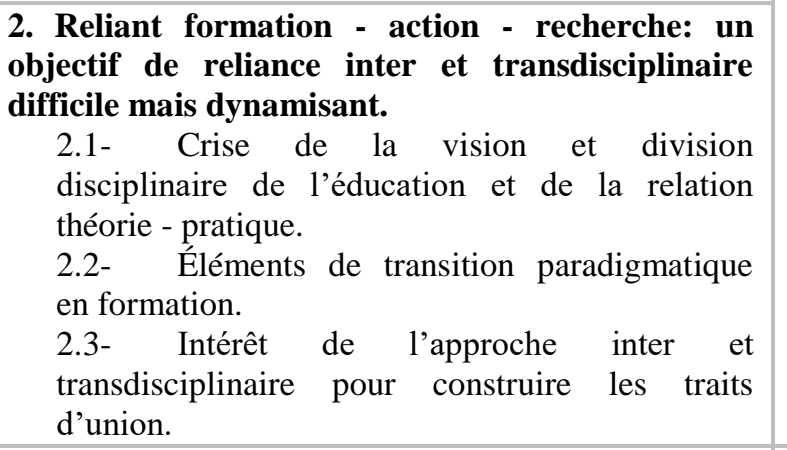 \\
\hline $\begin{array}{l}\text { 3. Par une pédagogie d'alternances coopératives } \\
\text { de production de savoirs. } \\
\text { 3.1- Alternances spatio-temporelles } \\
\text { bipolaires: pratiques / théories, action / réflexion, }\end{array}$ \\
\hline
\end{tabular}

\section{PRODUCTIONS LIEES AU MASTER}

UNEFAB，2002, «Alternância e Desenvolvimento sustentável », second Séminaire International, Brasília.

PINEAU, Gaston, 2004, «Temporalidades na formaçaõ», São Paulo, TRIOM dédié aux étudiants et à Pinheiro dos Santos (la rythmanalyse).

PUIG-CALVO, Pedro, 2003, «Les centres familiaux de formation par alternance: développement des personnes et de leurs milieux. Recherche-action internationale sur la place de la formation et de la recherche dans une institution», Université de Tours, Mémoire DURF. 
expériences / expressions.

3.2- Par apprentissage coopératif tripolaire: individu / société / environnement matériel.

3.3- De productions de savoirs: transformer

les connaissances expérientielles et les

informations bibliographiques en savoirs socialisables. a pratica no ambito da educação permanente» in Cadernos de Pesquisa em Educação, Universidade Federal do Espírito Santo, Vitoria, Volume 8, $\mathrm{n}^{\circ} 15$, p. $140-172$.

Mémoires de 17 acteurs - auteurs.

Selon: Pineau e Altri (2009).

L'ampleur des multicrises actuelles semble appeler à oser reprendre en compte ces coordonnées aussi basiques de «l'homme générique» que sont ces coordonnées spatio-temporelles. A la fin de son tome 5 de la Méthode sur l'identité humaine, Morin parle d'une seconde préhistoire. «Nous sommes dans une seconde préhistoire, celle de l'âge de fer planétaire, préhistoire d'une possible société-monde, et toujours préhistoire de l'esprit humain, peut-être préhistoire de l'ère technique» (Morin, 2001). De gré ou de force, nous sommes à l'orée d'une nouvelle ère planétaire. Une nouvelle planétarité est à construire avec de nouveaux devenirs se consolidant par superposition durable. Mais qu'est-ce que la durée? Un état continu à maintenir et reproduire ou des mouvements complexes à accorder?

Dans cette approche temporelle constructiviste de la formation humaine, le diplôme est heureux de renouer avec un philosophe brésilien pionnier mais méconnu. Il s'agit de Lucio Alberto Pinheiro dos Santos qui en 1931 a écrit une œuvre sur la rythmanalyse. Gaston Bachelard, en 1950, consacre le dernier chapitre de «La dialectique de la durée» (Bachelard, 1963) à ce terme en commentant cette œuvre par ailleurs introuvable. L'ouvrage «Temporalités en formation. Vers de nouveaux synchroniseurs» (Pineau, 2004) prolonge et actualise ces auteurs. Les grandes théories de la formation permanente sont exposées, ainsi que l'alternance et les récits de vie comme nouveaux synchroniseurs-donneurs de temps et de sens - de cette formation. Entre autres, la théorie tripolaire de la formation - par soi, les autres et les choses - déjà énoncée par Jean-Jacques Rousseau retrouve une actualité pertinente en fonction d'une tripolarité basique des devenirs.

En effet, ces bases spatio-temporelles de «devenirs durables» demeureraient assez inopérantes si elles ne servaient à étayer les trois piliers personnel, écologique et socio-économique qu'a heureusement introduit frontalement l'adoption - même idéologique - de la notion de développement durable. La 
conjugaison spatio-temporelle inter et transgénérationnelle de ces trois piliers est retenue comme horizon de recherche, d'action et de formation du diplôme.

\section{Une épistémologie transdisciplinaire pour relier formation-action et recherche}

$\mathrm{La}$ formation permanente et la recherche en formation est jeune : ses premiers indicateurs institutionnels d'existence datent des années soixante-dix. Apprendre à être (1972), rapport de la Commission internationale sur l'éducation de l'UNESCO, peut être mentionné comme une date de reconnaissance internationale. L'éducation permanente y est posée comme principe premier des politiques éducatives futures. Cette jeune recherche a d'abord émergé en marge, sinon en opposition et en réaction à une recherche en éducation beaucoup plus ancienne et déjà instituée selon un modèle classique déterminant de façon relativement précise le champ de la recherche éducative.

Dans cette transition paradigmatique, la jonction avec l'approche inter et surtout transdisciplinaire a été majeure pour construire des traits d'union entre ces opérations de recherche, d'action et de formation, clivées dans le paradigme disciplinaire classique. D'ailleurs le seul membre du groupe ne venant pas $\mathrm{du}$ mouvement de l'alternance, Américo
Somermman, était co-fondateur du premier Centre d'Éducation Transdisciplinaire (CETRANS) relié alors à l'université de Sao Paolo.

Tout d'abord, ce mouvement inter et transdisciplinaire n'est pas la négation des disciplines mais l'essai de leur utilisation selon des degrés d'ouverture et d'interaction variable. Ce mouvement est apparu dans les années 70 à la crête d'un mouvement de recherche de liens entre les disciplines pour contrebalancer un mouvement inverse de divisions disciplinaires proliférantes et inflationnistes. Face donc à une nécessité de spécialisation qui entraîne un émiettement des savoirs, il représente une autre nécessité de liaisons pour articuler des rationalités locales dans une rationalité d'ensemble. Sinon la contradiction entre les pôles locaux et globaux des développements fera éclater toute unité viable.

A cette nécessité disciplinaire interne de liens pluri, inter - et même transdisciplinaires s'ajoutent les pressions externes de problèmes qui ne se posent pas en terme ni disciplinaires, ni même disciplinés: problèmes de lutte contre la pauvreté, de pollution, d'emploi, de développement, de formation ... d'alternance Comment y répondre? L'université peut-elle longtemps demeurer 
absente de leur traitement parce qu'il ne relève directement d'aucune discipline? Et si indirectement ou partiellement plusieurs sont concernés, comment les articuler?

Cette pression externe transdisciplinaire est peut-être encore plus forte que les besoins internes de communication interdisciplinaire. C'est cette pression d'au-delà des disciplines qui a joué particulièrement pour transformer les problèmes d'actions, d'interactions et de transactions rencontrés dans les pratiques vécues d'alternance par les participants du master en projet de recherche pour les traiter. Et cette transformation, avec la production de savoirs qu'elle a produite, a constitué l'essentiel de la formation. Cette formation transdisciplinaire par production de savoirs situés, ancrés plus que par consommation de cours est l'objectif même du master.

\section{Une pédagogie d'alternances coopératives de production de savoirs}

Transformer des problèmes d'action en projet de recherche constitue donc une dynamique de formation qui met au second plan les logiques disciplinaires et les mobilise selon des conjugaisons variables intégrant au besoin d'autres sources de savoirs. Cette mobilisation et conjugaison ne peuvent se faire ni tout seul, ni en même temps, ni de manière uniforme. C'est pour ces opérations épistémologiques complexes que le diplôme fonctionne selon une pédagogie d'alternances coopératives de production de savoirs. Qu'est-ce à dire?

\section{L'alternance comme système interface entre pratique et théorie}

La bipolarisation de l'existence entre pratique et théorie semble bien être une structure anthropologique de base de la formation humaine, individuelle et collective. Le mythe de la caverne de Platon en présente un archétype toujours parlant.

Institutionnellement actuellement, cette bipolarisation a pris la forme d'un système travail et d'un système école aux logiques contradictoires (cf. tableau $\mathrm{n}^{\circ} 4$ ). Le premier poursuit une logique dominante de production subordonnant les savoirs à sa réalisation. Il faut d'abord réussir et cette réussite est une grande école expérientielle même si on ne peut pas toujours expliquer ni le pourquoi, ni le comment. La logique du système école estelle d'enseigner des savoirs formels et de développer la compréhension - en faisant l'hypothèse qu'elle suffit à la réussite.

Le défi est de reconnaître ces différences et ces oppositions tout en les rendant formatrices. De toute évidence l'écart est tel qu'un système interface est nécessaire pour faire communiquer, mettre 
ensemble et en sens ces opposés. Pas entièrement tout le temps, ce qui serait une fusion négatrice; mais par partie (dimension) et périodiquement, par alternance.
André Geay propose une alternance étude/travail selon un système interface à quatre dimensions: institutionnelle, didactique, pédagogique et personnelle, avec une ingénierie spécifique pour chacune (cf. tableau n ${ }^{\circ} 2$ ).

Tableau 3 - L'alternance comme système interface.

\begin{tabular}{|l|l|l|}
\hline \multicolumn{1}{|c|}{ Système Travail } & \multicolumn{1}{c|}{$\begin{array}{c}\text { Système interface à 4 } \\
\text { dimensions }\end{array}$} & \multicolumn{1}{c|}{ Système École } \\
\hline $\begin{array}{l}\text { Logique de production et } \\
\text { d'utilisation des savoirs. }\end{array}$ & INSTITUTIONNELLE & $\begin{array}{l}\text { Logique d'enseignement et } \\
\text { d'acquisition des savoirs. }\end{array}$ \\
\hline $\begin{array}{l}\text { Rapport finalisé au savoir (projet } \\
\text { professionnel). }\end{array}$ & DIDACTIQUE & $\begin{array}{l}\text { Rapport au savoir en soi (projet } \\
\text { d'études longues). }\end{array}$ \\
\hline $\begin{array}{l}\text { Stratégie d'apprentissage «réussir } \\
- \text { comprendre». }\end{array}$ & PEDAGOGIQUE & $\begin{array}{l}\text { Stratégies } \\
\text { «comprendre - réussir». }\end{array}$ \\
\hline Apprentissages expérientiels. & PERSONNELLE & Apprentissages formels. \\
\hline
\end{tabular}

Selon: Geay (1998).

La dimension institutionnelle de l'alternance suppose de développer un partenariat école-entreprise et une coresponsabilité de formation à parité d'estime. C'est le travail d'une ingénierie de projet de formations de compétences s'appuyant sur un référentiel d'entreprise contractualisé.
La dimension didactique appelle une démarche de formation inverse à la didactique scolaire classique. Elle part du métier avec concertation interdisciplinaire. Elle ne peut donc être mise en œuvre que par une ingénierie d'équipe pédagogique avec interventions alternées. 
La dimension pédagogique nécessite un partage de savoirs et du métier entre un enseignant et un maître d'apprentissage ou un tuteur. D'où une ingénierie d'exploitation de l'expérience et de suivi en double tutorat, par le tuteur mais aussi l'enseignant.

La dimension personnelle concerne l'autonomisation de l'alternant dans une démarche de production de savoirs et d'apprentissage de gestion de son temps par une ingénierie de la recherche et de l'autoformation.

Rapide et schématique est cette présentation de la conception et de la réalisation à temps et contretemps des différentes dimensions de ce système interface. Mais elle est suffisante pour pointer les transformations nécessaires des systèmes école et travail pour fonctionner de façon formative, c'est à dire distincte et complémentaire. Le livre d'André Geay (Geay, 1998) développe finement ces schémas.

\section{Le nécessaire partage partenarial et coopératif de la formation}

En anglais, on parle d'enseignement coopératif et non de formation en alternance. Si les deux expressions réfèrent à une même visée d'articulation entre travail et étude, l'expression anglaise de coopération ressort la dimension sociale d'opérations à faire au maximum ensemble, en partenariat; l'expression française alternance ressort la dimension temporelle. Ces opérations sociales d'étude et de travail se font dans des temps différents à conjuguer. Les deux expressions nous rappellent donc deux dimensions - temporelle et sociale - à l'œuvre dans ces formations mixant travail et études.

L'enseignement universitaire coopératif alternant implique que les représentants des organismes professionnels ne peuvent plus être considérés comme de simples opérateurs, de simples répondants ou correspondants pour encadrer les étudiants. Ils doivent devenir des co-opérateurs, des partenaires dans cette nouvelle production de nouveaux savoirs. Il ne s'agit pas seulement d'une nouvelle façon - un peu plus pratique et appliquée - de consommer des cours, des savoirs disciplinaires classiques. Il s'agit d'inventer, de construire institutionnellement de nouveaux rapports sociaux de production de savoirs. Nouveaux rapports sociaux où universitaires et professionnels sont vus comme producteurs de savoirs. Pas des mêmes savoirs. Ni de la même façon. Et c'est parce qu'ils sont différents que la coopération est nécessaire pour la production de savoirs complexes, 
nouveaux, pertinents pour traiter les problèmes nouveaux posés par un monde mouvant, mobile, en mouvement perpétuel.

Des recherches sur les conditions partenariales d'une formation alternante co-opérative, productrice de savoirs, Carol Landry, dresse une typologie des partenaires de l'alternances (Landry, 2002).

- Le partenaire producteur entrepreneur;

- Le partenaire patron - employeur;

- Le partenaire philanthrope;

- Le partenaire professionnel formateur.

Le partenaire producteurentrepreneur est d'abord mobilisé par la production à assurer selon une logique économique où l'alternance permet une sélection des meilleurs candidats. Il entretient des relations privilégiées, surtout formelles et fonctionnelles, avec les partenaires, principalement administratifs, de l'école.

Les objectifs du partenaire patronemployeur dépasse les horizons de sélection immédiate pour viser le contrôle maximal des dispositifs de l'alternance selon un logique politico-économique d'alternance-marketing. Ses relations principales sont de type institutionnel et stratégique avec les «gros» partenaires sociaux impliqués: état et syndicats.
Le philanthrope, lui voit l'alternance d'abord comme un moyen d'aide à l'insertion professionnelle des jeunes. Dans cet objectif et cette logique, il entretient des relations personnelles avec l'alternant et les partenaires pédagogiques.

Le partenaire professionnelformateur se centre sur l'efficacité de la formation. Il en discute professionnellement avec l'alternant et les partenaires pédagogiques en travaillant l'intégration de l'apport expérientiel de ce temps terrain dans le processus d'ensemble de la formation.

Cette typologie des partenariats possibles qu'implique l'institutionnalisation de la formation universitaire en alternance montre que cette formation ne soulève pas seulement des problèmes pédagogiques internes aux universités. Elle soulève aussi des problèmes, écologiques, de construction de nouveaux rapports entre l'université et ses environnements et même encore plus globalement entre économie et éducation.

La troisième partie de l'ouvrage coordonné par Landry étudie l'institutionnalisation de ces formations en alternance d'un point de vue macrosocial, dans la recherche de nouveaux rapports entre éducation et économie. Cette approche éducative est indissociable de perspectives inter-institutionnelles et socio- 
économiques. Cette troisième partie commence par poser des balises théoriques extrêmement intéressantes pour approcher ce méga-problème des rapports entre éducation et économie posé par l'alternance. A côté des grosses approches structurelles, P. Doray et B. Fusulier par exemple, optent pour une approche des négociations constituantes qui permet de saisir plus finement l'évolution des politiques et des stratégies d'acteurs dans la construction sociale de l'alternance. Et avec cette approche, ils analysent de façon comparatives deux dispositifs d'alternance au Québec et en Belgique.

\section{La production de savoirs par articulations d'informations et de connaissances}

Cette multi-diversité sociale et spatio-temporelle ne peut être unifiée de façon formatrice que par le seul acteur de base vivant tous les éléments de cette diversité: l'alternant. Lui seul peut conjuguer de façon formative à la première personne du singulier les apports parfois contradictoires des multiples personnes, espaces et temps rencontrés, affrontés. Le statut professionnel salarié de l'alternant adulte peut aider à rompre avec l'attitude souvent trop passive, trop dépendante de l'enseignant, induite par le statut scolaire du jeune élève ou étudiant classique. De plus l'ouverture sociale de l'alternance à des espaces/temps de travail productif appelle des stratégies d'apprentissage inverses de celles liés à l'enseignement. L'alternant ne doit pas seulement écouter assis, et tenter de comprendre. Il doit faire, circuler, se tenir debout, se déplacer, se poser, s'exposer, réussir ses actions. L'expérience est une redoutable maîtresse: elle fait passer l'examen d'abord et donne les leçons ensuite. D’abord faire, réussir. Ensuite comprendre. L'apprenant alternant n'est plus toujours lié physiquement à l'enseignant. Il doit apprendre à apprendre sans lui, avec d'autres, avec lui-même. L'alternance pour être formatrice appelle une autonomisation de l'apprentissage hors enseignement. Elle appelle même un renversement des rapports: l'enseignement au service de l'apprentissage et non l'inverse. Paradoxalement le trans et l'inter ne sont formateurs qu'en développant l'auto. C'est en s'auto-disciplinant, en développant ses capacités autonomes d'apprentissage que l'alternant apprend à produire du savoir avec et au-delà des disciplines, avec d'autres et même contre eux. Pour assurer cette production personnelle de savoirs, l'alternant doit acquérir un statut de partenaire d'acteurchercheur-auteur.

Cette autonomisation cognitive, éminemment auto-formative, réinterroge la nature du savoir. Elle oblige à voir le 
cognitif et sa construction de façon plus différenciée, transdisciplinaire, à prendre en compte l'information transmise par les médias multiples et aussi les connaissances acquises par les acteurs à l'école de leur vie, entre autres, professionnelle. $\mathrm{La}$ trilogie savoir-connaissance-information dans le prolongement de Dewey (Legroux, 2008) se révèle pédagogiquement très éclairante et fructueuse.

La connaissance, comme le porte son étymologie, est un cognitif incorporé à l'acteur. Elle fait corps avec lui. Est le produit de ses expériences, de ses interactions organisme - environnement. Ce qu'il fait qu'il n'est pas un idiot culturel mais au contraire un être intelligent, réflexif, parlant, pilotant son devenir. Mais ce cognitif fait tellement partie de la vie de l'acteur, qu'il a du mal à se formuler et encore plus à se formaliser. Il est en grande partie insu, tacite, pré-conscien L'information à l'opposé, est une unité cognitive autonome, pouvant même changée de support médiatique-orale, écrit, visuel, électronique. Elle est flottante.

Le savoir est le cognitif échangeable, interface entre les deux: de la connaissance formulée et formalisée ou de l'information organisée et structurée.

Produire du savoir nécessite de se brancher au moins sur l'une ou l'autre de ces sources, au mieux sur les deux en les dosant de manière optimale. $\mathrm{Ne}$ se connecter qu'à des ouvrages porteurs d'informations multiples c'est produire un savoir apparemment objectif et universel mais décontextualisé. Rester obstinément et exclusivement attaché à ses sources expérientielles, c'est produire un savoir très subjectif, peu échangeable et communicable. D'où la nécessité pédagogique, pour transformer les acteurs sociaux en auteurs de savoirs universitaires et professionnels - d'alterner, de combiner et de confronter expressions personnelles et empiriques avec prise d'informations bibliographiques, analyses et synthèses.

Une opération est centrale pour cette perlaboration cognitive: écrire. Centrale mais difficile. Aussi pour cette production de savoirs, la première stratégie pédagogique est de jalonner le parcours de production d'écrits. Dès la première session, il est demandé à chacun, de résumer l'idée de projet de recherche en une phrase: première production. La deuxième session, on a demandé de produire cinq pages pour préciser le projet sur tel objet, pour tel objectif, avec tel public, avec telle méthode. A la fin de la première année: 30 pages pour synthétiser le travail de l'année et préparer le travail de la seconde année. A la sixième et à la septième session, nous avons voulu des écrits de synthèse sur deux modules: le 
module développement durable et le module formation pour que les étudiants puissent se consacrer ensuite uniquement sur la production du mémoire final.

Cette nécessité de production se retrouve certainement dans d'autres mestrados. Celui-ci essaie de favoriser au maximum cette obligation en croisant autoproduction et co-production. A chaque session, 4 ou 5 acteurs en apprentissage d'auteurs doivent présenter leur projet. Les présentations orales mutualisent et socialisent l'état de ces savoirs mais aussi leur processus de construction. En plus, ces présentations collectives sont accompagnées d'entretiens individuels, de travail personnel et à la demande, d'interventions plus systématiques, type cours ou conférences.

\section{Les productions classées selon le modèle des quatre piliers de l'alternance}

D'une recherche-action internationale très liée à celle de la démarche du master Formation et Développement Durable, Pedro PuigCalvó coordonnateur pédagogique du master, ressort quatre piliers structurant la base sociale associative, coopérative et partenariale de l'alternance éducative (cf. tableau $\left.n^{\circ} 6\right)$.

Une organisation participative, l'Association locale de base, dont la responsabilité directe, la gestion et les projets incombent principalement aux familles, mais aussi aux communautés, aux institutions locales, aux professionnels, aux promoteurs et aux personnes associées. Une méthodologie éducative propre, l'Alternance intégrative, basée sur la distribution des périodes de formation entre l'école et le milieu familial (socioprofessionnel), à savoir l'entreprise familiale ou une autre entreprise. L'interaction éducative entre l'école et le milieu est la constante de ce système. Une formation intégrale de l'élève en tant que personne; une formation professionnelle, intellectuelle, humaine, sociale, morale, spirituelle qui permet de dessiner son propre projet de vie, avec un accent mis sur le projet professionnel personnel et, si possible, à partir et au sein du milieu d'où il est issu. Le développement local au travers de l'éducation des jeunes et des adultes appelés à devenir les véritables acteurs du progrès de leur milieu. (Puig-Calvó, Les centres familiaux de formation par alternance: développement des personnes et des milieux, 2003). 
Tableau $\mathrm{n}^{\circ} 4$ - Les quatre piliers des Centres Familiaux et Formation par Alternance (CEFFA).

\section{Les 4 piliers des CEFFA}

Centres éducatifs familiaux de formation par alternance

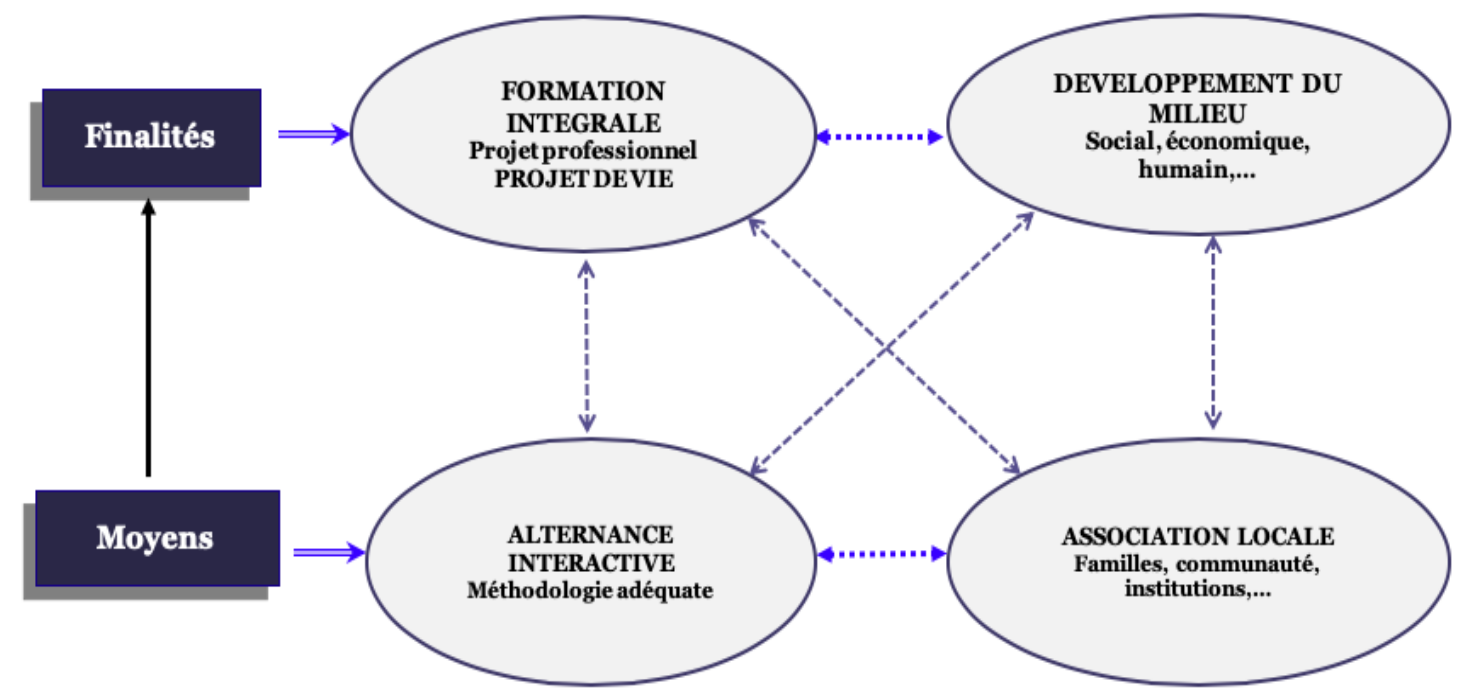

Selon: Puig-Calvó, 2003. (Puig-Calvó, 2019).

Cette structure à quatre piliers peut rendre compte des recherches conduites par les participants du Mestrado «formation et développement durable» $\mathrm{du}$ Brésil (2002-2004). Les thèmes des recherches mettent l'accent sur l'un ou l'autre des quatre piliers sans perdre de vue l'appréhension globale de la formation en lien avec chaque contexte spécifique. Deux thèses présentées en final prolongent la structure quaternaire en interrogeant les savoirs nécessaires pour le futur et les transformations paradigmatiques à travailler. À partir simplement de leur titre comme indicateur de leur orientation majeure, les 15 autres mémoires vont être distribués selon cette structure quadripolaire, la concrétisant et l'ancrant dans des pratiques précises de recherche formation à partir des problèmes d'action rencontrées. Neuf se centrent sur les finalités (Formation intégrale de la personne et développement du milieu), et six sur les modalités (pédagogie et association). Répartition donc relativement équilibrée.

\section{Formation intégrale de la personne}

$$
\text { Cinq mémoires travaillent }
$$
frontalement cette finalité de formation intégrale de la personne. Celui de Isabel Xavier de Oliveira Rocha l'affiche nommément dans son intitulé: Uma reflexão sobre a formação integral nas 
EFAs. Contributos do Caderno da Realidade nesse processo na EFA de Riacho de Santana - Bahia. Deux autres l'explore pour des personnes jeunes: Formação de jovens e participação social: Um estudo sobre a formação de três jovens da Escola Família Agrícola de Porto Nacional-TO. (Erialdo Augusto Pereira); et Identidade do Jovem Rural: Confrontando com Estereótipo de Jeca Tatu: Um estudo qualitativo com os jovens da EFA Rei Alberto I (Antônio Carlos Frossard). Et un autre plus à long terme, dans l'itinéraire de vie d'un agriculteur pour soupeser le poids respectif de la formation formelle et expérientielle dans cet itinéraire: Formação formal e experiencial: estudo de fatores-chave no itinérario de um agricultor. (Agostinho Barrionuevo).

Le cinquième le fait à partir de l'apprentissage de la temporalité au moyen de L'enseignement de l'histoire: $O$ ensino de história e a aprendizagem das temporalidades (Gilede Cardoso Pereira).

\section{Développement du milieu socio- économique, humain et politique}

Cette deuxième finalité de l'alternance mobilise aussi la rechercheformation de quatre mémoires. Deux l'affichent aussi dans leur titre même: $A$ escola de ensino fundamental rural no desenvolvimento do seu meio: um estudo comparativo. (Francisco Trevisan); Contribuição da Escola Família Agrícola de Riacho de Santana para o desenvolvimento do meio: um estudo com os Ex-Alunos (David Rodrigues de Moura de Riacho de Santana). Les deux autres l'aborde dans le mouvement d'évolution agricole: $\quad D a$ agricultura tradicional à agricultura familiar inovadora: contributo das formações no rito de passagem. (Antonio Locatelli); avec les problèmes de financement et précarité économique: $A$ Precariedade do financiamento $e$ os desafios: O caso de duas Escolas Famílias Agrícolas em uma região de acentuada pobreza no Brasil. (Mário Sebastião Cordeiro Alves).

\section{La méthodologie pédagogique de l'alternance}

Deux titres affichent cette méthodologie pédagogique comme objectif de leur recherche-formation: Formação pedagógica de monitores das escolas famílias agrícolas e alternâncias: Um Estudo Intensivo dos Processos Formativos de cinco Monitores (João Batista Begnami); et Formação de lideranças e pedagogia da alternância: um estudo do itinerário de três jovens reconhecidos como líderes (Márcio Andrade). Les deux autres l'abordent sous un angle plus précis: le plan de formation, 

mouvements et le master formation et développement durable au Brésil...

$O$ plano de formação na alternância e o processo de ensino-aprendizagem: Um Estudo na Escola Família Agrícola de Goiás. (Ana Maia Pereira Pinto); et la construction de projet politique, Processo de construção de projeto político pedagógico - Um estudo na EFA do PACUI. (Hildete Margarida R. de Souza).

\section{Organisation participative}

La participation du milieu socioprofessionnelle est exploré par Thierry de Burghrave à partir de l'histoire de vie de deux agriculteurs Autoformação $e$ participação no meio sócio-profissional: abordagem biográfica de dois agricultores do Movimento das Escolas Famílias Agrícola. Et celle de la famille par Sergio Zamberlan: O lugar da família na vida institucional da escola-família: Participação e Relações de Poder. Agostinho Barrionuevo.

\section{Évolution paradigmatique du savoir et éducation du futur}

Deux mémoires débordent cette structure quadripolaire de l'alternance pour la situer plus largement dans l'évolution paradigmatique des savoirs et des perspectives d'éducation du futur: Formação e transdisiplinaridade: uma pesquisa sobre as emergências formativas do Cetrans. (Américo Sommerman). Novo paradigma educativo e práticas pedagógicas das EFA's - Análise de planos de estudo inovadores em relação aos sete saberes de Morin. (Rachel Reis Menezes).

Chaque auteur de mémoire en a fait une synthèse d'une vingtaine de pages, constituant 17 chapitres sur 19 de l'ouvrage collectif qui est sorti en français en 2009: Alternatives socio-éducatives au Brésil. Expérience d'un master international.

Une visée ample et à long terme: produire des savoirs reliant les trois devenirs, personnel, social et écologique

Viser à construire des devenirs durables a donc amené à tenter d'articuler au mieux la recherche à l'action en concevant et pilotant une formation selon une pédagogie d'alternances coopératives basée sur l'apprentissage accompagné de production d'écrits, comme stratégie de production de savoirs. Sur les 20 possibles, 17 mémoires ont été produits.

Mais la production de savoirs jugés pertinents au plan universitaire n'est pas suffisante en soi pour assurer des devenir durables. Elle est sans doute nécessaire mais ne peut se déployer durablement, économiquement, écologiquement et personnellement, que si elle s'accompagne d'une formation optimale des producteurs eux-mêmes, comme humains, 
professionnels et terriens. Cette formation optimale d'acteurs-auteurs concrétise les finalités de formation intégrale et de développement du milieu poursuivies par l'alternance associative.

Les savoirs en effet peuvent autant délier, diviser, opposer que relier, conjoindre, articuler. Cette ambivalence fait de leur production un enjeu politique et stratégique majeur pour construire les liens entre formation et devenirs durables, de personnes de sociétés et de planète. C'est cet enjeu que travaille la philosophie socioéducative de ce diplôme dans son fonctionnement même. En effet, cette philosophie reste lettre morte si elle n'est pas portée par une ingénierie partenariale de fonctionnement, entre institutions universitaires et professionnelles mais aussi entre les acteurs de la formation.

Assurer ce double fonctionnement partenarial - interinstitutionnel et interactoriel - est fondamental. Difficile, il demande du temps, de l'énergie, de l'engagement éthique responsable et autonome ainsi qu'un apprentissage stratégique organisationnel constant, ferme et ouvert. Des ententes interinstitutionnelles ont été négociées et ont cadré les grandes lignes du fonctionnement partenarial. Une commission pédagogique exécutive très soudée en équipe de recherche-action- formation a piloté de très près la conception et réalisation du diplôme. Mais seul l'avenir à moyen et long terme confirmera si cette pédagogie d'alternances coopératives visant à articuler de façon inter et transdisciplinaire la formation à l'action par la recherche, a pu produire des savoirs anthropoformatifs, reliant les devenirs personnels, socio-économiques et écologiques.

\section{Conclusion: S'initier à la rythmo- formation}

Cinquante ans et même quatre-vingt ans, ce n'est pas si long pour apprendre à changer de régime et passer d'une religion de l'horaire et d'un temps uniforme à respecter, à l'initiation aux rythmes cosmiques pour danser sa vie, dans son intimité, avec celle des autres et celle du monde C'est avec ces rythmes qu'il faut apprendre à résonner (Rosa, 2018) sans se laisser arraisonner par tous les cours et discours de la bourse, des parlements, des maître et contremaîtres.

Puissent ces deux petites histoires de vie d'initiation à l'alternance, entre narration expérientielle et mise en forme conceptuelle, aider à l'apprentissage de ces résonnances, à cette rythmoformation. $\mathrm{La}$ compétence rythmique est une compétence temporelle générique existentielle, qui dépasse toute profession et toute discipline 
(Pineau, 2018-2019) En résonance au premier théoricien de la rythmanalyse, Lucio Alberto Pinheiro dos Santos, Gaston Bachelard nous a appris que «Il faut guérir l'âme souffrante ... par une vie rythmique, par une pensée rythmique, par une attention et un repos rythmique ... le rythme est vraiment la seule manière de discipliner et de préserver les énergies les plus diverses» (Bachelard, 1963). Lucio est né à Braga en 1889 et mort à Rio de Janeiro en 1950 après avoir entre autres enseigné à Carangola dans le Minas Gerais en 1927. Fêter cette année 2019, les 130 ans de sa naissance et l'an prochain les 70 ans de sa mort, serait le re-susciter et nous avec.

\section{Références}

Landry, C. (2002). La formation en alternance. État des pratiques et des recherches. Montreal: Presses de l'Université de Quebec.

Carré Ph., Moisan A., \& Poisson, D. (2010). L'autoformation. Perspectives de recherche. Paris: PUF.

Chartier, D. (2004). À l'aube des formations par alternance. Paris: l'Harmattan.

Legroux, J. (2008). De l'information à la connaissance. Paris: L'Harmattan.

Lesourd, F. (2009). L'homme en transition. Éducationet tournants de vie. Paris: Anthropos.
Bachelard, G. (1963). La dialectique de la durée. Paris: PUF.

Eneau, J. (2005). La part d'autrui dan la formation de soi. Autonomie, autoformation et réciprocité en contexte organisationnel. Paris: L'Harmattan.

Gadotti, M. (2000). Pedagogia da Terra. Sao Paulo: Petropolis.

Geay, A. (1998). L'école de l'alternance. Paris: l'Harmattan.

Moneyron, A. (2018). Agroécologie. Quelle écoformation? Paris: L'Harmattan.

Morin, E. (2001). La méthode 5. L'identité humaine. Paris: Seuil.

Morin, E. (2002). Au-delà de la globalisation et du développement, societémonde ou empire-monde? Revue $d u$ Mauss, 43-52.

Pineau. (2015). De l'air Essai sur l'ecoformation humaine. Paris: l'Harmattan.

Pineau G., Bacherart D., Couceiro L., Gimonet, J. C., \& Puig-Calvó, P. (2009). Alternatives socio-éducatives au Brésil. Expérience d'un master international. Paris: L'Harmattan.

Pineau, G. (1977). Ëducation ou alienation permanente? Repères mythiques et politiques. Montreal: Dunod.

Pineau, G. (2004). Temporalidades na formaçao. Rumbo a novos sincronizadores. TRIOM: Sao Paulo.

Pineau, G. (2017). Un nouvel âge pour l'Alternance. Présences, 83-88.

Pineau, G. (2018-2019). Conjuger les temporalités pour en faire des rythmes formateurs. Éducation Permanente, (4), 921. 
Pineau, G., \& Galvani, P. (2017). Exploration de l'ecoformation humaine avec les quatre éléments. Dans L. Sauvé, Éducation/Environement/Écocytoyenneté. Répère contemporains. Montreal: Presses de l'Université du Quebec.

Puig-Calvó, P. (2003). Les centres familiaux de formation par alternance: développement des personnes et des milieux. Tours: Universoté de Tours (Memoire DURF).

Puig-Calvó, P. (2019). Formation des Formateurs PROCEFFA. Yaoundé (Cameroun): AIMFR.

Reinberg, A., \& Ghata J. (1982). Les rythmes biologiques. Paris: PUF.

Rosa, H. (2018). Resonance.Une socilogie de la realtion au Monde. Paris: La découverte.

Rousseau, J. J. (2009). L'Émile ou De l'éducation. Paris: Flammarion.

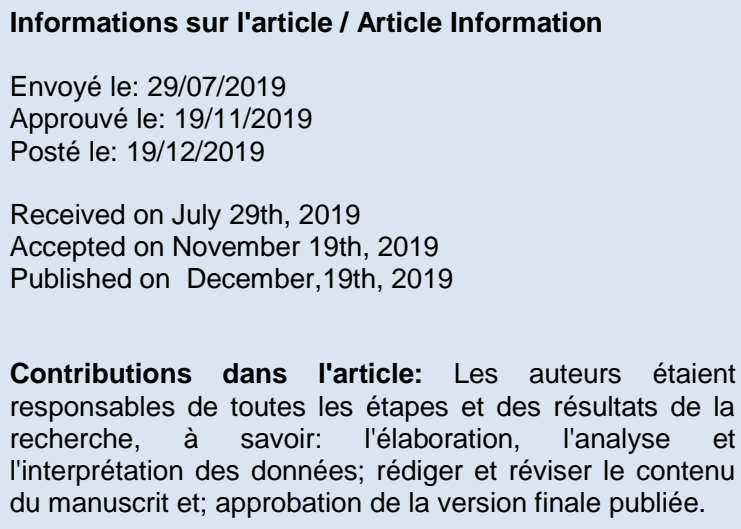
responsables de toutes les étapes et des résultats de la recherche, à savoir: l'élaboration, l'analyse et l'interprétation des données; rédiger et réviser le contenu du manuscrit et; approbation de la version finale publiée.

Author Contributions: The author were responsible for the designing, delineating, analyzing and interpreting the data, production of the manuscript, critical revision of the content and approval of the final version published.

Conflits d'intérêts: Les auteurs ont déclaré qu'il n'y avait pas de conflit d'intérêts concernant cet article.

Conflict of Interest: None reported.

Orcid

Gaston Pineau

iD http://orcid.org/0000-0002-0060-0669

Pedro Puig-Calvó

(iD) http://orcid.org/0000-0002-1367-6478

Comment citer cet article / How to cite this article

APA

Pineau, G., \& Puig-Calvó, P. (2019). Histoires de vie avec l'alternance: la voie de recherche-formation en deux temps trois mouvements et le master formation et développement durable au Brésil. Rev. Bras. Educ. Camp., 4, e7279. DOI: http://dx.doi.org/10.20873/uft.rbec.e7279

ABNT

PINEAU, G.; PUIG-CALVO, P. Histoires de vie avec l'alternance: la voie de recherche-formation en deux temps trois mouvements et le master formation et développement durable au Brésil. Rev. Bras. Educ. Camp., Tocantinópolis, v. 4, e7279, 2019. DOI: http://dx.doi.org/10.20873/uft.rbec.e7279 